\title{
The Influence of Benoît B. Mandelbrot on Mathematics
}

\author{
Edited by Michael F. Barnsley and Michael Frame
}

\section{Michael F. Barnsley}

\section{Introduction}

We begin this article, which deals largely with Benoît B. Mandelbrot's contributions to and influence upon mathematics, with a quotation from the introduction to Fractals: Form, Chance, and Dimension [16]. This essay, together with many pictures and numerous lectures in the same vein, changed the way science looks at nature and had a significant impact on mathematics. It is easy for us now to think that what he says is obvious; it was not.

Many important spatial patterns of Nature are either irregular or fragmented to such an extreme degree that Euclid-a term used in this essay to denote all classical geometryis hardly of any help in describing their form. The coastline of a typical oceanic island, to take an example, is neither straight, nor circular, nor elliptic, and no other classical curve can serve, without undue artificiality in the presentation and organization of empirical measurements and in the search for explanations. Similarly, no surface in Euclid represents adequately the boundaries of clouds or rough turbulent wakes....

Michael F. Barnsley is a professor at the Mathematical Sciences Institute, Australian National University. His email address is Michae1. Barns1ey@anu. edu . au.

Michael Frame is adjunct professor of mathematics at Yale University. His email address is mi chae1 . frame@ya 1e . edu.

DOI: http://dx.doi.org/10.1090/noti894
In the present Essay I hope to show that it is possible in many cases to remedy this absence of geometric representation by using a family of shapes I propose to call fractals - or fractal sets. The most useful among them involve chance, and their irregularities are statistical in nature. A central role is played in this study by the concept of fractal (or Hausdorff-Besicovitch) dimension....Some fractal sets are curves, others are surfaces, still others are clouds of disconnected points, and yet others are so oddly shaped that there are no good terms for them in either the sciences or the arts. The variety of these forms should be sampled by browsing through the illustrations....

$$
\text { -Benoît B. Mandelbrot [16, pp. 1-2] }
$$

As with the now familiar principle that gravitational force tethers the earth to the sun, it has become hard to imagine what it was like not to know that many physical phenomena can be described using nondifferentiable, rough mathematical objects.

Important fractals such as the Cantor set, the Sierpinski triangle, and Julia sets were well known to some mathematicians, but they were neither visible nor promoted to any practical purpose. To me, looking back, it seems that these beautiful things were hidden behind veils of words and symbols with few diagrams, certainly no detailed pictures; for example, the long text (in French) of Gaston Julia failed to reveal to most people, including most mathematicians, the full wonder of 
the endless arabesques and intricate visual adventures in the boundaries of Fatou domains. It was as though such objects were guarded by the priests of mathematics, occasionally to be displayed, like the monstrance at Benediction, to the inner core of true believers. I was ritually inducted to calculus in my first year at Oxford by Hammersley, who took us through a full proof of the existence of a Weierstrass nowhere differentiable continuous curve from first principles. Half an hour with pictures would have saved a lot of time and would not have tainted our logical skills.

Benoît not only wrested these abstract objects, these contrary children of pure mathematics, out from the texts where they lay hidden, but he also named them and put them to work to help to describe the physical observable world. He saw a close kinship between the needs of pure mathematics and the Greek mythological being Antaeus. In an interview [6] Benoît said, "The son of Earth, he had to touch the ground every so often in order to reestablish contact with his Mother, otherwise his strength waned. To strangle him, Hercules simply held him off the ground. Separation from any down-to-earth input could safely be complete for long periods-but not forever." He also said, "My efforts over the years had been successful to the extent, to take an example, that fractals made many mathematicians learn a lot about physics, biology, and economics. Unfortunately, most were beginning to feel they had learned enough to last for the rest of their lives. They remained mathematicians, had been changed by considering the new problems I raised, but largely went their own way."

John Hutchinson is an example of a pure mathematician who was strongly influenced by Benoît's work.

In 1979 I was on study leave from the Australian National University, visiting Fred Almgren at Princeton for 6 months, as a result of my then interest in geometric measure theory. While there, Fred suggested I read Mandelbrot's book Fractals: Form, Chance and Dimension and look at putting it, or some of it, into a unified mathematical framework. As a result, we organised a seminar in which I spoke about six times as my ideas developed. Participants included, besides Fred and myself, Bob Kohn, Vladimir Schaeffer, Bruce Solomon, Jean Taylor and Brian White. Out of this came my 1981 article "Fractals and self-similarity" [7] in the Indiana University Math. Journal, which introduced the idea of an iterated function system (though not with that name) for generating fractal sets, similar ideas for fractal measures, and

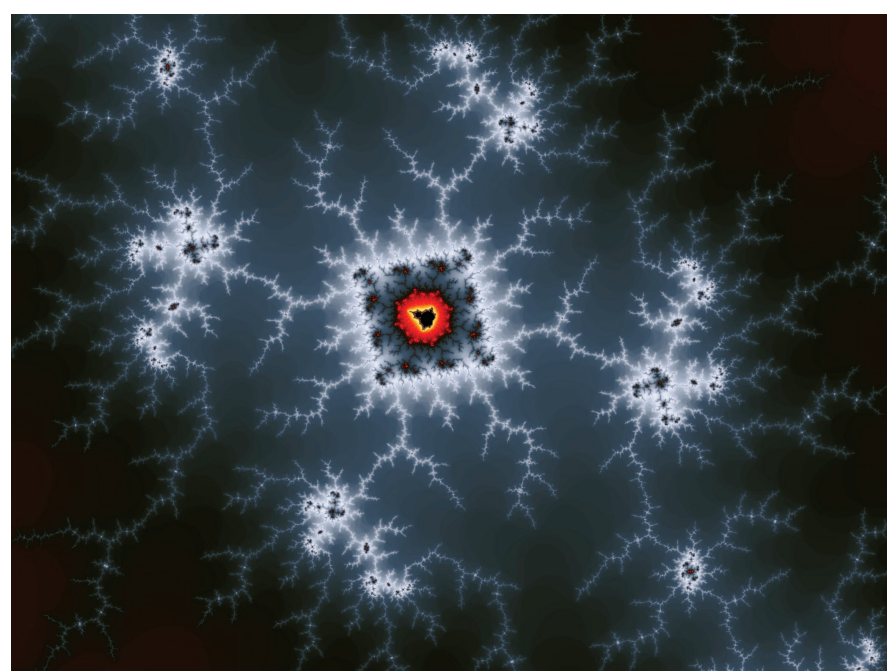

Figure 1. An outlier Mandelbrot set (M-set) (surrounded by yellow, then red) connected via a branch of a tree-like path to the whole M-set. The connectivity of the M-set was conjectured by Benoît in 1980 and established by Adrien Douady and John Hubbard in 1982.

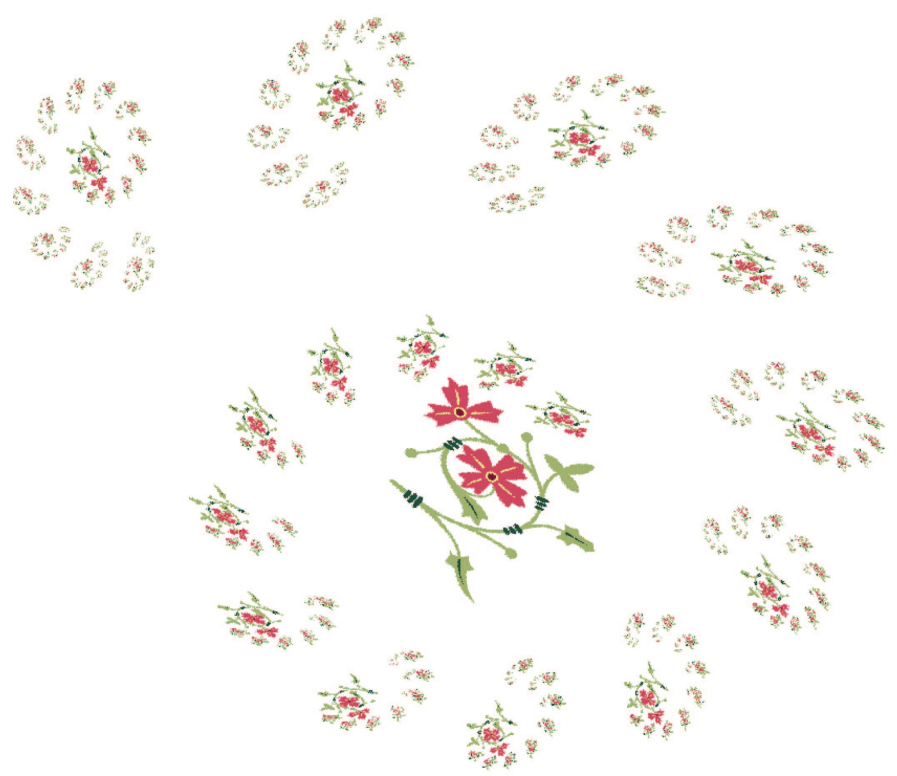

Figure 2. Picture of $\mathcal{F}^{16}(S)$ where $S \subset \mathbb{R}^{2}$, $\mathcal{F}(S)=f_{1}(S) \cup f_{2}(S)$, and $f_{1}, f_{2}: \mathbb{R}^{2} \rightarrow \mathbb{R}^{2}$ are affine contractions. The sequence $\left(\mathcal{F}^{n}(S)\right)$ converges in the Hausdorff metric to a self-similar set, a fractal, with Hausdorff dimension less than two. This article has been decorated with pictures, in the spirit of Benoît.

various structure theorems for fractals. Interestingly, this paper had no citations for a few years, but now it frequently gets in the AMS annual top ten list.

Mandelbrot's ideas were absolutely essential and fundamental for my paper. I still 
have my original copy of his book, signed by Benoît, on the one occasion at Princeton that we met.

\section{-John Hutchinson}

Iterated function systems (IFSs) are now a standard framework for handling deterministic fractals, self-similar sets and measures. They were named by this author and Stephen Demko [1], though Benoît thought we should have called them "map bags". He was fascinated by models of leaves with veinlike internal structures made by invariant measures of IFSs.

Hutchinson's paper and the work of many others influenced by Mandelbrot ended a long period where geometry and the use of pictures played little role in mathematics. Mandelbrot believed passionately in pictorial thinking to aid in the development of conjectures and formal proofs. His advocacy has enabled it to be okay once again for mathematicians to do experimental mathematics using pictures.

Mandelbrot's ideas have inspired a huge amount of research, from pure mathematics to engineering, and have resulted in deep theorems; a new acceptance of geometry and pictures as having a role to play in experimental mathematics; and various applications, including image compression and antenna design. The notion of a fractal now forms part of good preuniversity mathematics education, while the mathematical study of fractals has its own specialist areas, including, for example, analysis on fractals [8] and noncommutative fractal geometry [9].

One important idea of Mandelbrot was that various random phenomena, such as stock market prices, are governed by probability distributions with "fat tails". This led him to warn in 2004 that "Financial risks are much underestimated. I think we should take a strongly conservative attitude towards evaluating risks." The subsequent global financial crisis underlined his point.

Prior to editing both this article and [3], we emailed colleagues to ask for memories and comments on Benoît's contributions to mathematics, influence, and personal recollections. We received replies from many: not only mathematicians but artists, physicists, biologists, engineers, and so on. Using these replies we have produced two articles: this one and [3], which is more focused on recollections of the man himself. Our goal has been to put together something special using the words of everyone who wrote but, in general, editing and shortening to avoid repetition of themes.

From early on, Mandelbrot was driven by a desire to do something totally original, to look at problems that others found too messy to consider, and to find some deep unifying principles. As the

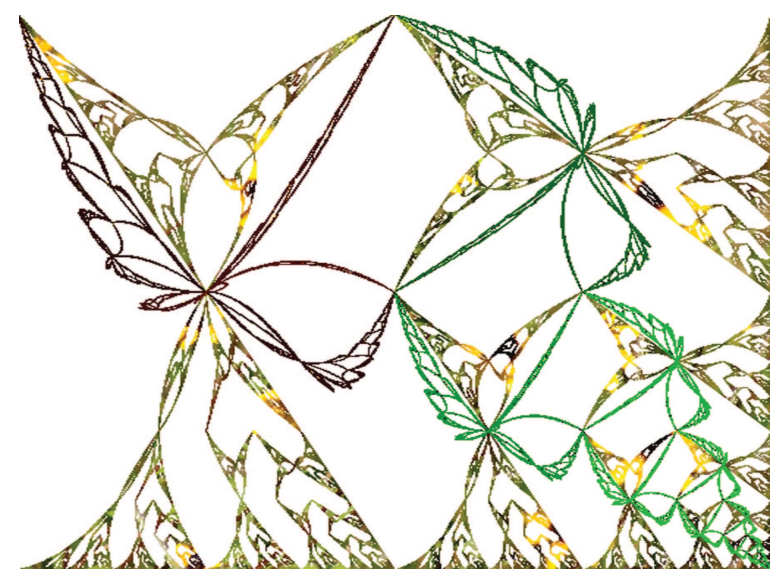

Figure 3. Superposition of the attractors, colored using fractal transformations (see [2]) of two simple bi-affine iterated function systems.

words in the following contributions show, he succeeded.

\section{Roger Howe}

\section{Participating in a Conversation That Takes Place over Long Spans of Time}

One pleasure of doing mathematics is the sense of participating in a conversation that takes place over long spans of time with some of the smartest people who ever lived. Benoît's work on fractals provides a good example of this kind of long-term dialogue.

A significant factor in the invention of calculus was the idea of representing a curve by the graph of a function and, reciprocally, of representing the time variation of a quantity by a curve. This backand-forth identification allowed one to connect the drawing of tangent lines with finding the rate of change of quantities that vary in time.

When calculus was invented in the seventeenth century, the concept of function was not very precise. Work during the eighteenth century on solving the wave equation using sums of sine and cosine functions led to a sharpening of understanding of the essential properties of functions and of their behavior. This led in the first half of the nineteenth century to the isolation by Cauchy of the notion of continuity, which made clear for the first time the distinction between continuity and differentiability. During the rest of the nineteenth century, mathematicians explored this difference, which contributed to the general unease and insecurity about the foundations of mathematics. Hermite is quoted as "recoiling in horror from functions with no derivatives." The early twentieth century saw the production of

Roger Howe is professor of mathematics at Yale University. His email address is roger.e. howe@ya le.edu. 

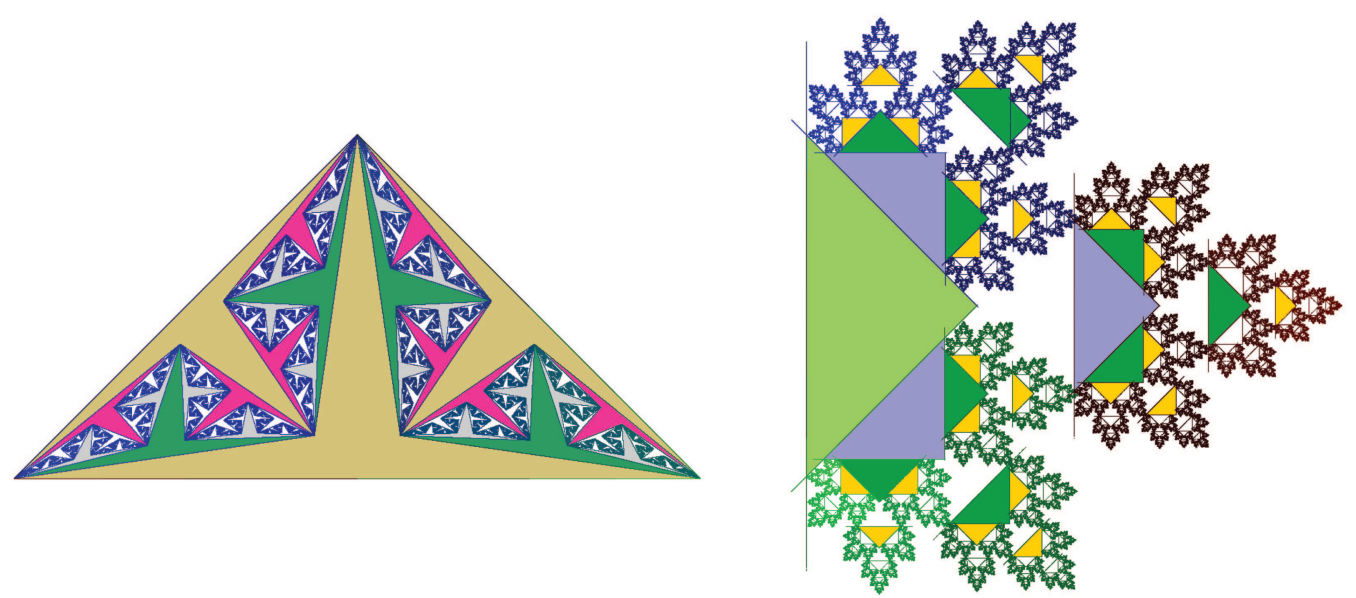

Figure 4. Two illustrations of IFS semigroup tilings. The triangle on the left is tiled with the orbit of a six-sided figure under a system of two affine transformations. The limit set of the set of triangular tiles on the right is the attractor of a system of three affine transformations. A theme of Benoît's work was that the iteration of simple rules (e.g., elementary geometrical transformations) can produce nondifferentiable (rough) objects. Figure from [2].

a menagerie of striking examples (the Cantor set, the Koch snowflake, the Sierpinski carpet, etc.) illustrating the difference between continuity and differentiability. However, for several decades these examples were regarded as exotica, monsters with no relation to the physical world. They were objects only a mathematician would investigate. They were liberated from this marginal status by Mandelbrot, who said, "Wait a minute. A lot of things in the world-clouds, river systems, coastlines, our lungs - are well described by these monsters." Thus started the use of these mathematical objects to study complicated, messy nature.

\section{Ian Stewart}

\section{No Lily-White Hands}

I first learned about fractals from Martin Gardener's Scientific American column. I promptly bought a copy of Fractals: Form, Chance, and Dimension [16]. Despite, or possibly because of, its unorthodoxy and scope, it seemed to me that Benoît Mandelbrot had put his finger on a brilliant idea.

I'm pleased that, towards the end of his life, he received due recognition, because it took a long time for the mathematical community to understand something that must have been obvious to him: fractals were important. They were a game changer, opening up completely new ways to think about many aspects of the natural world. But for a long time it was not difficult to find professional

Ian Stewart is emeritus professor of mathematics at the University of Warwick, UK. His email address is I.N. Stewart@warwick.ac.uk. research mathematicians who stoutly maintained that fractals and chaos were completely useless and that all of the interest in them was pure hype. This attitude persisted into the current century, when fractals had been around for at least twentyfive years and chaos for forty. That this attitude was narrow-minded and unimaginative is easy to establish, because by that time both areas were being routinely used in branches of science ranging from astrophysics to zoology. It was clear that the critics hadn't deigned to sully their lily-white hands by picking up a random copy of Nature or Science and finding out what was in it.

To be sure, Mandelbrot was not a conventional academic mathematician, and his vision often carried him into realms of speculation. And it was easy to maintain that he didn't really do much that was truly novel-fractal dimension had been invented by Hausdorff, the snowflake curve was a century old, and so on. Mathematicians would have cheerfully gone on employing HausdorffBesicovitch dimension to consider such questions as finding a set of zero dimension that covers every polygon, but they would not have figured out that quantifying roughness would make it possible to apply that kind of geometry to clouds, river basins, or how trees damp down the energy of a hurricane.

Mandelbrot's greatest strength was his instinct for unification. He was the first person to realize that, scattered around the research literature, often in obscure sources, were the germs of a coherent framework that would allow mathematical models to go beyond the smooth geometry of manifolds, a reflex assumption in most areas, and tackle the irregularities of the natural world 


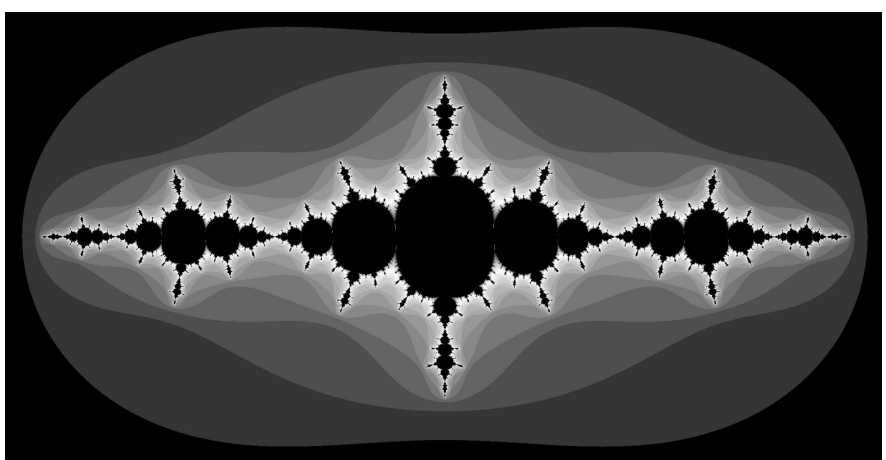

Figure 5. A Julia set associated with the first cascade of period doubling bifurcations of the logistic equation. Julia sets for quadratic maps are intimately related to the Mandelbrot set. Benoît was one of the first to use computers to make pictures of mathematical objects: computations which took hours to run on expensive mainframes can now be performed in seconds on handheld devices. This image and Figures 1 and 13 were computed using free software (Fractile Plus) on an iPad.

in a systematic fashion. It took many years before these ideas began to pay off, but that's how pioneering mathematics often goes.

The conjecture whose proof so pleased him (see [3, contribution by Ian Stewart]) was the work of Gregory Lawler, Oded Schramm, and Wendelin Werner in their paper "The dimension of the planar Brownian Frontier is 4/3" [10]. It is part of the work for which Werner received a Fields Medal, and it shows that fractals have given rise to some very deep mathematics. I suspect that only now are we beginning to see the true legacy of Mandelbrot's ideas, with a new generation of researchers that has grown up to consider chaos and fractals to be as reasonable and natural as periodic motion and manifolds. Mandelbrot was a true pioneer, one of the greatest mathematical visionaries of the twentieth and early twenty-first centuries.

\section{David Mumford}

\section{Benoît Told Me: "Now You Can See These Groups and See Teichmüller Space!"}

Benoît Mandelbrot had two major iconoclastic themes. First, that most of the naturally occurring measurements of the world were best modeled by nondifferentiable functions, and second, the histograms of these measurements were best modeled by heavy-tailed distributions. Even if he did not bring a new unifying law like Newton's

David Mumford is emeritus professor of mathematics at Brown University. His email address is DavidMumford@ brown. edu.
$F=m a$ and even if he did not have the deep and subtle theorems that make waves in the pure math community, this vision was revolutionary. What his lectures made clear was that fractal behavior and outlier events were everywhere around us, that we needed to take these not as exceptions but as the norm. For example, my own work in vision led me later on to express his ideas about outliers in this way: that the converse of the central limit theorem is true, namely, the only naturally occurring normal distributions are ones which are averages of many independent effects.

Benoît's immediate effect on my work was to reopen my eyes to the pleasure and mathematical insights derived from computation. I had played with relay-based computers in high school and with analog computer simulations of nuclear reactors in two summer jobs. But at the time I thought that only white-coated professionals could handle the IBM mainframes and puzzled over what in heaven's name my colleague Garrett Birkhoff meant when I read " $x=x+1$ " in some of his discarded code. But Benoît told us that complex iterations did amazing things that had to be seen to be believed. These came in two types: the limiting behavior of iterations of a single analytic function and the limiting behavior of discrete groups of Möbius transformations. The second of these connected immediately to my interests. I was always alert to whatever new tool might be available for shedding any sort of light on moduli spaces, whether it was algebro-geometric, topological, characteristic p point counting, or complex analytic. I had sat at the feet of Ahlfors and Bers and learned about Kleinian groups and how they led to Teichmüller spaces and hence to moduli spaces. Benoît told me, "Now you can see these groups and see Teichmüller space!"

I found an ally in Dave Wright, learned C, and with Benoît's encouragement, we were off and running. When he returned to his position at the IBM Watson Lab, he set up a joint project with us, and we visited him and his team there. Later, Curt McMullen, who also appreciated the power and insight derived from these experiments, joined us. It turned out that, in the early hours of the morning, their mainframes had cycles to spare, and we would stagger in each morning to see what these behemoths had churned out. There was no way to publish such experiments then, but Dave and I astonished the summer school at Bowdoin with a live demo on a very primitive machine of a curvy twisting green line as it traced the limit point set of a quasi-Fuchsian group. Ultimately, we followed Benoît's lead in his Fractal Geometry of Nature [18] and, with Caroline Series, published our images in a semipopular book, Indra's Pearls [27]. One anecdote: We liked to analyze our figures, 
estimating, for example, their Hausdorff dimension. We brought one figure we especially liked to Watson Labs and, thinking to test Benoît, asked him what he thought its Hausdorff dimension was. If memory serves, he said, "About 1.8", and indeed we had found something like 1.82. He was indeed an expert!

\section{Hillel Furstenberg}

\section{He Changed Fundamentally the Paradigm with Which Geometers Looked at Space}

Let me begin with some words of encouragement to you on this project, dedicated to memorializing an outstanding scientist of our times and one we can be proud of having known personally.

What do you see as Benoit's most important contributions to mathematics, mathematical sciences, education, and mathematical culture?

Benoît Mandelbrot sold fractals to mathematicians, changing fundamentally the paradigm with which geometers looked at space. Incorporating fractals into mainstream mathematics rather than regarding them as freakish objects will certainly continue to inspire the many-sided research that has already come into being.

\section{Kenneth Falconer}

\section{It Was Only on the Fourth or Fifth Occasion That I Really Started to Appreciate What He Was Saying}

Benoît's greatest achievement was that he changed the way that scientists view objects and phenomena, both in mathematics and in nature. His extraordinary insight was fundamental to this, but a large part of the battle was getting his ideas accepted by the community. Once this barrier was broken down, there was an explosion of activity, with fractals identified and analyzed everywhere across mathematics, the sciences (physical and biological), and the social sciences.

Benoît realized that the conventional scientific and mathematical approach was not fitted to working with highly irregular phenomena. He appreciated that some of the mathematics needed was there-such as the tools introduced by Hausdorff, Minkowski, and Besicovitch-but was only being used in an esoteric way to analyze specific pathological sets and functions, mainly as

Hillel Furstenberg is professor of mathematics at BarIlan University, Israel. His email address is harry@math. huji.ac. $i 1$.

Kenneth Falconer is professor of pure mathematics at the University of St. Andrews in Scotland. His email address is kjf@st-andrews.ac.uk.

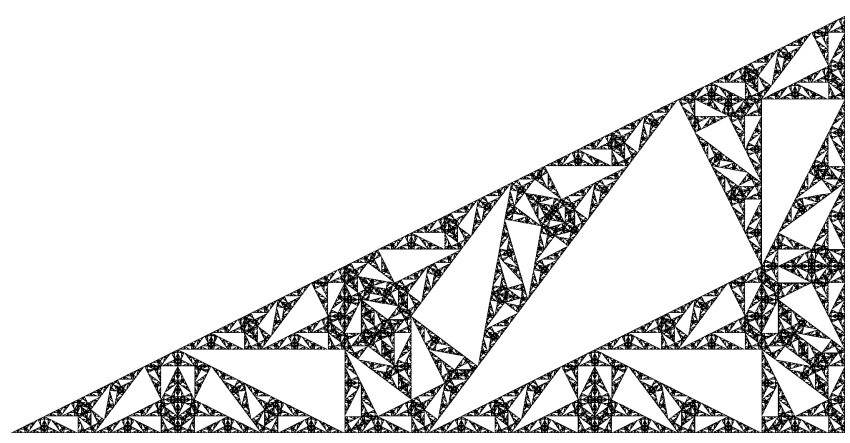

Figure 6. A self-similar fractal of Hausdorff dimension $(4 \ln 2) / \ln 5 \doteq 1.72$ associated with the pinwheel tiling.

counterexamples that illustrated the importance of smoothness in classical mathematics.

Benoît's philosophy that such "fractal" objects are typical rather than exceptional was revolutionary when proposed. Moreover, he argued that the mathematical and scientific method could and should be adapted to study vast classes of fractals in a unified manner. This was no longer mathematics for its own sake, but mathematics appropriate for studying all kinds of irregular phenomena-clouds, forests, surfaces, share prices, etc.-that had been ignored to a large extent because the tools of classical smooth mathematics were inapplicable.

Benoît also realized that self-similarity, broadly interpreted, was fundamental in the genesis, description, and analysis of fractals and fractal phenomena. Given self-similarity, the notion of dimension is unavoidable, and "fractal dimension" in various guises rapidly became the basic measure of fractality, fuelling a new interest in the early mathematics of Hausdorff, Minkowski, and others.

Benoît had many original ideas, but his presentation of them did not always follow conventional mathematical or scientific styles, and as a result it often took time for his ideas to be understood and sometimes even longer for them to be accepted. A case in point is that of multifractal measures. Multifractals are, in many ways, more fundamental than fractal sets. Many of the now standard notions of multifractals may be found in his 1974 paper in the Journal of Fluid Dynamics [14], but this is not an easy paper to fathom, and it was not until the 1980s that the theory started to be appreciated. Benoît suggested that "the community was not yet ready for the concept," but I think the delay was partly because of the way the ideas were presented. I heard Benoît's talk on multifractals many times in the 1980s; he was charismatic, but his explanations were such that it was only on the fourth or fifth occasion that I really started to appreciate what he was saying. 


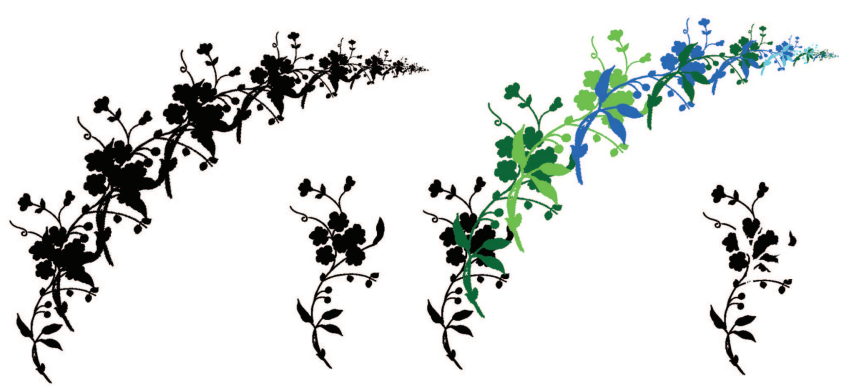

Figure 7. The left-hand picture illustrates the points in the orbit of a set; the flower picture at center left, under a Möbius transformation. The picture at center right reveals that it is a "tiling", where the initial tile is shown on the right. Mandelbrot caused many to look anew at natural objects in geometrical terms. Figure from [2].

I am one of many whose life and career have been influenced enormously by Benoît and his work, both directly and indirectly. We miss him, but the legacy of his ideas and work will remain with us all and with those who follow.

\section{Bruce J. West}

\section{The Intermittent Distribution of the Stars in the Heavens}

Benoît's idiosyncratic method of communicating mathematical ideas was both challenging and refreshing. The introduction of geometrical and statistical fractals into the scientific lexicon opened up a new way of viewing nature for a generation of scientists and allowed them to understand complexity and scaling in everything from surface waves on the ocean to the irregular beating of the heart to the sequencing of DNA. This accelerated the early research done by biologists, physicians, and physicists on the understanding of complex phenomena.

The line between what was proven and what was conjecture in Benoît's work was often obscure to me, but in spite of that, or maybe even because of that lack of clarity, I was drawn into discussions on how to apply the mathematics of fractals to complex phenomena. Fractals began as descriptive measures of static objects, but dynamic fractals were eventually used to describe complex dynamic phenomena that eluded description by traditional differential equations. Culturally, fractals formed the bridge between the analytic functions of the nineteenth- and twentieth-century physics of acoustics, diffusion, wave propagation, and quantum mechanics to the

Bruce J. West is adjunct professor of physics at Duke University. His email address is bruce . $j$. west@us . army . mi 1. twenty-first-century physics of anomalous diffusion, fractional differential equations, fractional stochastic equations, and complex networks.

Benoît identified some common features of complex phenomena and gave them mathematical expression without relying on the underlying mechanisms. I used this approach to extract the general properties of physiological time series, which eventually led to the formation of a new field of medical investigation called Fractal Physiology, the title of a book [28] I coauthored in 1995 and the subject of an award-winning book [29] on the fractional calculus. Later, in 2010, I became founding editor-in-chief of the new journal Frontiers in Fractal Physiology, which recognizes the importance of fractal concepts in human physiology and medicine.

I first met Benoît when I was a graduate student in physics at the University of Rochester. Elliott Montroll, who had the Einstein Chair in Physics and who had been a vice president for research at IBM, was friends with Benoît and would invite him to come and give physics colloquia. In the late 1960s, before the birth of fractals, I heard Benoît conjecture as to why the night sky was not uniformly illuminated because of the intermittent distribution of stars in the heavens, why the price of corn did not move smoothly in the market but changed erratically, and why the time between messages on a telephone trunkline were not Poisson distributed as everyone had assumed. These problems and others like them struck me as much more interesting than calculating perturbation expansions of a nuclear potential. So I switched fields and became a postdoctoral researcher in statistical physics with Elliott. I have interacted with many remarkable scientists, and Benoît is at the top of that list. I am quite sure that my decision to change fields was based in large part on Mandelbrot's presentations and the subsequent discussion with him and Montroll.

\section{Marc-Olivier Coppens}

\section{Engineering Complexity By Applying Recursive Rules}

As a chemical engineering researcher who worked with Benoît since the middle of the 1990s, I benefited a lot from his mentorship. I also miss him a lot as a friend. In 1996, while completing my Ph.D. thesis, I worked closely with him for several months at Yale, sharing an office with Michael Frame. I developed, with Benoît, a new way to

Marc-Olivier Coppens is professor and associate director of the Multiscale Science and Engineering Center, Rensselaer Polytechnic Institute. His email address is coppens@rpi.edu. 


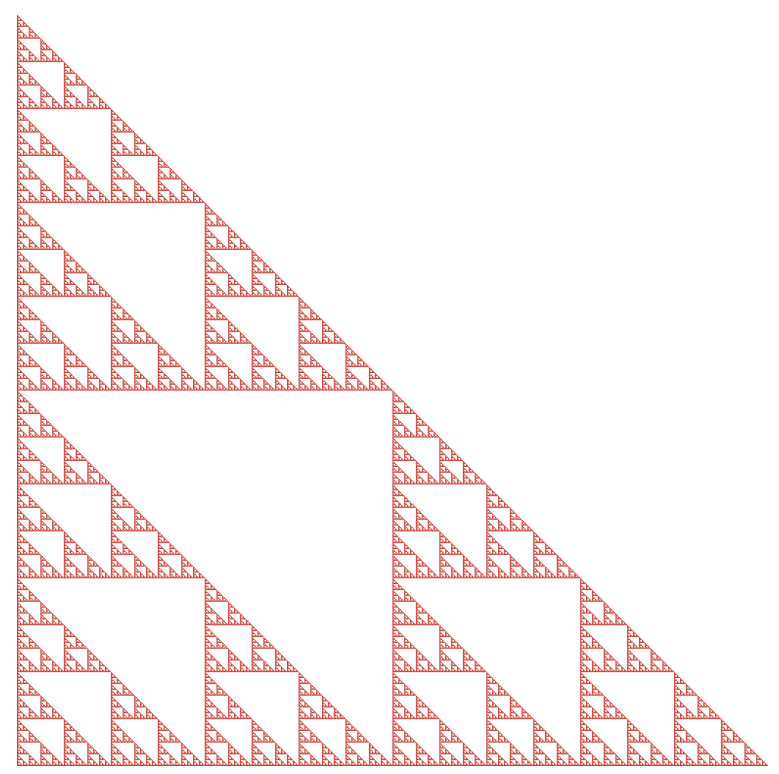

Figure 8. A right-angle Sierpinski triangle. Benoît realized that such objects were not freaks and belonged in mainstream mathematics. Analysis on fractals is now a fascinating area of mathematics.

generate multifractals by taking the product of harmonics of periodically extended functions.

Fractals in chemical engineering have affected the modeling and characterization of various porous materials. As Mandelbrot liked to say in later years, fractals are an ideal way to measure "roughness", and roughness is prevalent in chemical engineering and materials science. The roughness of porous media affects transport and reactions in them and hence has a significant impact on chemical engineering. For example, in my thesis I showed how molecular-scale roughness of porous catalysts influences chemical product distributions up to industrial scales.

In my research I have used fractal trees to interpolate efficiently between the micro- and the macroscale, as in nature. Scaling up from the laboratory to the production scale requires preservation of small-scale, controlled features up to larger scales. This challenge is met by distributing or collecting fluid in a uniform way, as is realized by scaling fractal architectures in nature, such as trees, lungs, kidneys, and the vascular network. Specifically, I proposed a fractal, treelike injector to uniformly distribute fluids over a reactor volume, so that the fluids can mix and interact with the reactor contents. This patented fractal injector has proven very efficient for gassolid fluidized beds. My laboratory is currently developing a fractal fuel cell design, inspired by the structure of the lung.

Benoît has had a major influence on my thinking. To a large extent, thanks or due to the advance

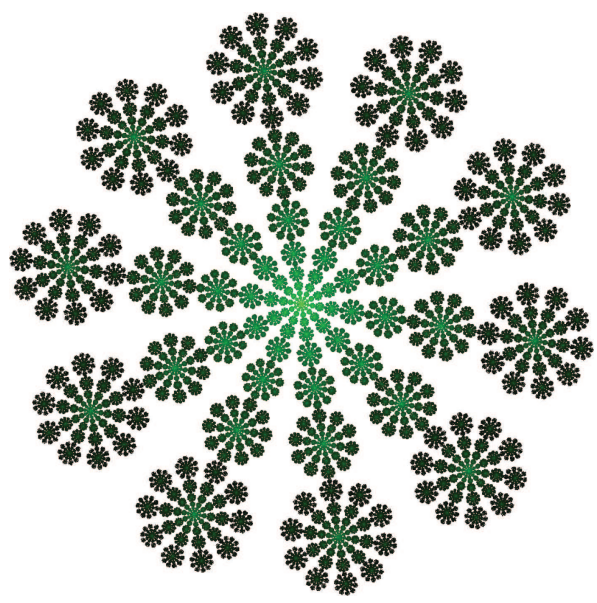

Figure 9. An invariant measure on a fractal attractor of a system of three similitudes has here been rendered in shades of green. (Bright green = greater "density", black=least "density".)

of massively parallel, high-performance computers, chemical, biological, and materials sciences are increasingly atomistic, deconstructing and constructing matter out of individual elements in which the details of each component and its interactions are more and more explicitly accounted for. This atomistic treatment is very powerful and facilitates the study of specific properties of matter. However, sometimes the importance of the forest tends to be lost in looking too closely at one tree. The complementary, holistic view is, in my opinion, extremely powerful as well, as it allows us to see essential features in a phenomenon without the need to resolve every detail. Fractals are an example of this idea, where complexity emerges from the combination of simple rules. A marriage between the holistic and atomistic views can lead us beyond the deficiencies of each one separately.

\section{Nathan Cohen}

\section{Complexity Was Well Modeled by Fractals}

Mathematicians spar in an uncomfortable match between the pure and applied, in which migration from one to the other is one way, and no one is allowed to do both. But Benoît Mandelbrot did.

My interest in fractals stems from needing to solve real-world problems. In 1985 I was a newly minted Ph.D. in Cambridge (MA). There the general view was that fractals were a "flavor" of the month, and they were treated as an a posteriori paradigm with no evidence of solving problems unsolved in other ways. But I read The Fractal Geometry

Nathan Cohen is the founder of Fractal Antenna Systems, Waltham, Massachusetts. His email address is ncohen@ fractenna.com. 


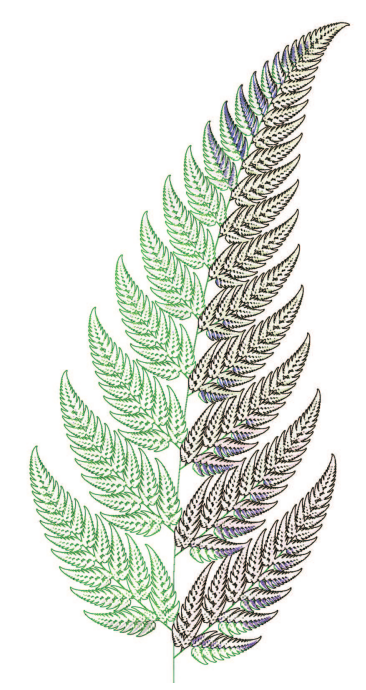

Figure 10. A self-affine fractal provides a simple model for the geometry of a fern.

of Nature and landed a consulting job on stock options pricing. I concluded, as Mandelbrot had surmised decades earlier, that the stock price is not a "random walk", that complexity and noise are often indistinguishable, and that complexity may be modeled by fractals. Market pricing is essentially deterministic, not random. At that time, on a daily basis, traders would run their Black-Scholes models, which assume pricing is a random diffusion process, and bring the results to the floor each morning like racing forms at the horsetrack. They trusted these cheat-sheets to tell them when to buy and sell. But I was able to exploit the limitations of the Black-Scholes model using fractals and made a decent little fortune for someone who had recently been a poverty-stricken student.

The notion of "fractals as antennas" occurred to me in 1987 while attending a lecture by Mandelbrot. I went home and explored this curious idea, which has subsequently become a major theme of my efforts and a field in its own right. Some years later I saw Benoît again at a fractalsin-engineering conference. This was finally the opportunity to converse with him and the first of several lunch meetings and subsequent phone conversations in the last dozen years of his life. No one who had such conversations can forget the brilliant, witty joy of Benoît the polymath. In particular, they helped me to realize that Maxwell's equations require self-similarity for frequency invariance, a fundamental and what should have been obvious result. Now I see many problems that benefit from fractals: metamaterials, a new form of radiative transport, optimization, and fluid mechanics and drag reduction. I only regret that I can't share these with Benoît anymore.
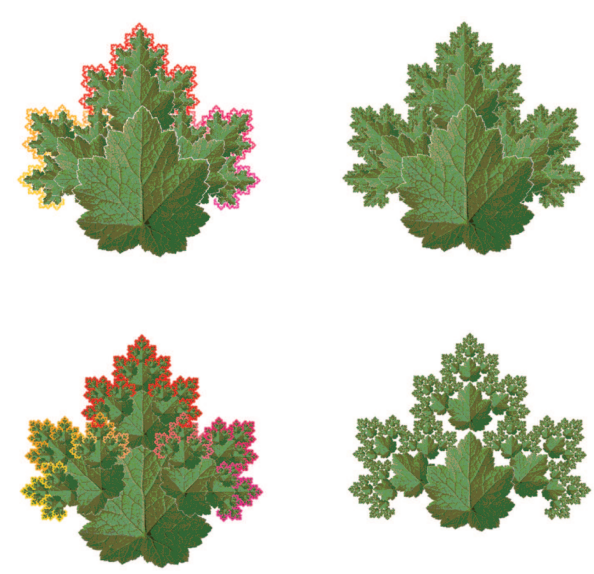

Figure 11. Various pictures constructed from the orbit of a leaf picture under a system of three affine transformations. The limit set of the semigroup is illustrated in red and yellow. Figure from [2].

\section{Stéphane Jaffard}

\section{Parts of Mathematics Are Totally Bathing in the Ideas That Benoît Introduced}

Benoît was one of the first to apply computer graphics to mathematical objects. He used them to develop intuitions and to make either discoveries or deep conjectures.

He also put forward particular entities such as Mandelbrot cascades, the Mandelbrot set, Lévy dusts, and so on as beautiful objects, worthy of study in their own right. At that time, this was orthogonal to the main direction of mathematics towards generalizations and abstract structures. I believe that Benoît's influence on the mathematical community was very helpful in that respect: mathematics was able to admit a down-to-earth component. Some parts of mathematics are now totally bathing in the ideas that Benoît introduced. For example, the idea of scale invariance is everywhere present in the mathematics of signal processing, my area.

More broadly, the notion of fractal probability has been one of the most important unifying concepts in science introduced in the last fifty years. It has allowed scientists with diverse specializations to draw connections between seemingly unrelated subjects and has created unexpected cross-fertilizations. This was driven by the mesmerizing and enthusiastic personality of Benoît.

Note that fractals are one of the few parts of mathematics that can be "shown" to the general

Stéphane Jaffard is professor of mathematics at Université Paris Est (Créteil Val-de-Marne). His email address is stephane.jaffard@u-pec.fr. 


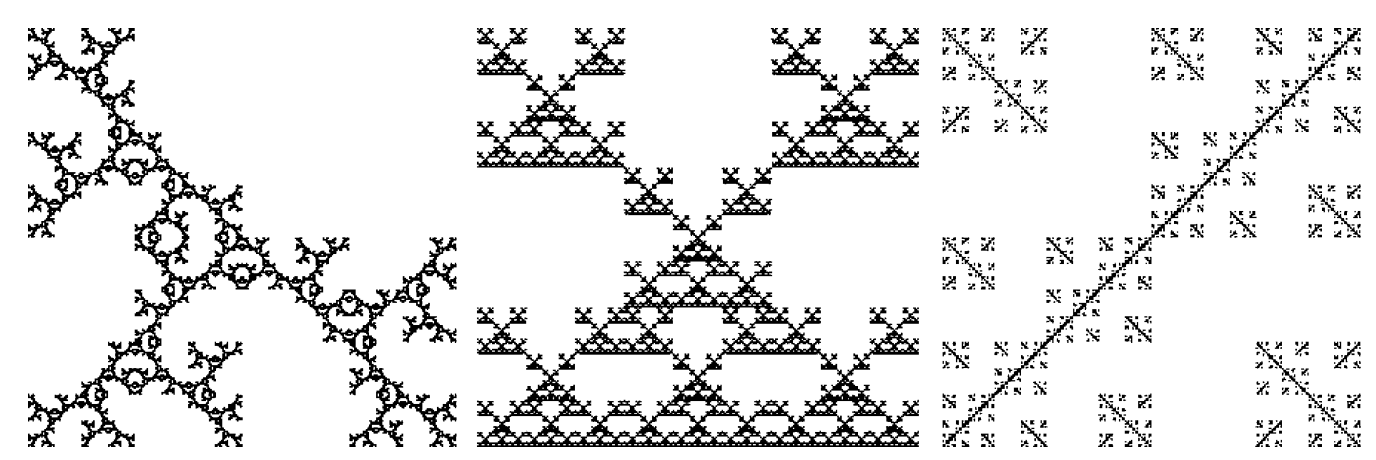

Figure 12. “...[E]ighty students in my fractal geometry course learn in a single class how to generate the fractals pictured here...."

public. As a teenager, I was influenced by Benoît's fascinating books. They explained a part of mathematics that was under construction yet could be readily understood.

My thesis was on the then-new topic of "wavelets". I worked at École Polytechnique under the supervision of Yves Meyer. Once Benoît visited École Polytechnique, and he heard that a Ph.D. student was working on systems of functions that could be decomposed into elementary blocks, related inter alia by dilations and translations. He came to my office, and we had long conversations about new possibilities offered by wavelet analysis. For me, this was the start of interactions which influenced me considerably; it certainly pushed me towards specializing in multifractal analysis, a part of fractals where Mandelbrot's ideas are prevalent. Our interactions resulted in two joint papers on Polya's function, whose graph is space-filling and multifractal (its Lipschitz regularity index jumps everywhere). The interest that Benoît showed in this example, which was quite forgotten at that time, was typical of his fascination for beautiful mathematical objects and the art with which he managed to draw a correspondence between their mathematical beauty and their graphical beauty. In all the conversations that we shared, I was always amazed by the uninterrupted flow of original and brilliant ideas that he very generously shared.

\section{Sir Michael Berry}

\section{How to Model...a Surface With No Separation of Scales}

In the early 1970s, I was studying radio-wave echoes from the land beneath the ice in Antarctica. Existing theories separated the "geography", supposedly measured by the start of the echo, from the "roughness", indicated by the disorderly

Sir Michael Berry is professor of physics at the University of Bristol, UK. His email address is asymptotico@ physics.bristol.ac.uk. echo trail. The separation was modelled by a flat surface ("geography") superimposed on what was single-scale randomness ("roughness"), typically gaussian. I found this not only unappealing but also scientifically absurd: in a natural landscape, any apparent dichotomy must be an illusion, an artifact of the wavelength used to interrogate it. But how to model, or even describe, a surface with no separation of scales? I had no idea until I read Philip Morrison's review of the English edition of Mandelbrot's Fractals: Form, Chance and Dimension [16]. I cannot remember being so excited by a book review. It was immediately clear that fractal dimension was the key idea I needed, and this was confirmed by the book itself.

Quickly came the identification of a new class of wave phenomena: "diffractals", that is, waves interacting with fractal objects. In the echo-sounding of landscapes, the interaction is mainly reflection. Later, a grim consequence of an absorption interaction emerged: we realized that the prolonged winter predicted to occur after a nuclear war, because of the absorption of sunlight by smoke, would be significantly intensified by the fact that smoke particles are fractal (it would also be prolonged, because smoke's fractality slows the particles' fall). From the development of quantum chaology in the late 1970s came a conjecture about the spectra of enclosures ("drums") with fractal boundaries: the "surface" correction to the "bulk" Weyl eigenvalue counting formula would scale differently with frequency and depend on the fractal dimension. This generated considerable mathematical activity.

In diffractals it is the objects interacting with the waves, not the waves themselves, that are fractal. But in some phenomena the wave intensity is fractal on a wide range of scales down to the wavelength. One such, unexpected in one hundred fifty years, is the Talbot effect, associated with light beyond diffraction gratings whose rulings have sharp edges: the fractal dimensions of the wave across and along the beam direction are different. All this sprang from Benoît Mandelbrot's insight, 


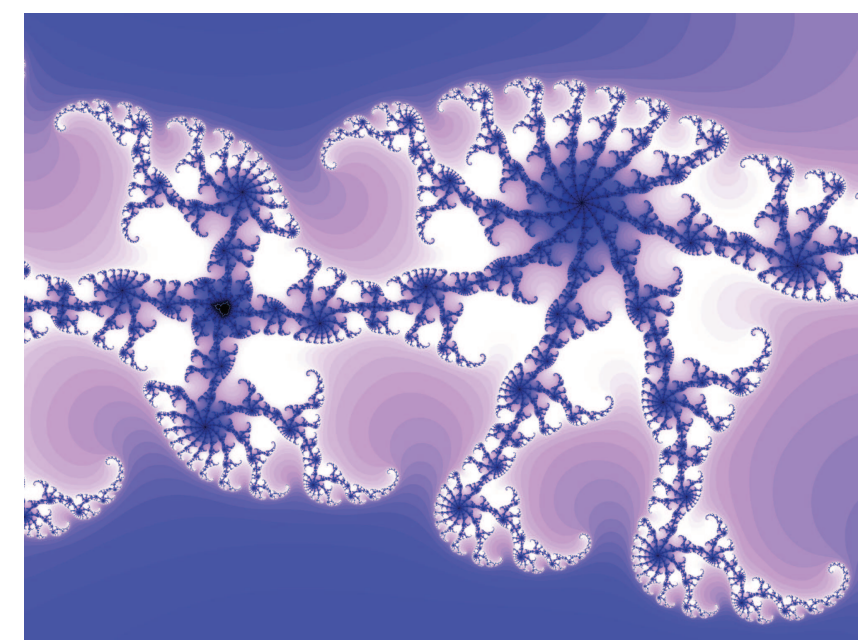

Figure 13. "Zoom in a few times...mysterious spirals of spirals of spirals appear."

meshing perfectly with my preoccupations at the time. For further details, see my earlier tribute [4] or my home page [5].

\section{Michael Frame}

\section{Believe the Classroom Is an Appropriate Stage for a Final View of Benoît's Work}

Here I'll give a sketch of the remarkable breadth and depth of Benoît's work, setting most examples in the world I know best, the classroom. That students in college, high school, and elementary school study the concepts Benoît developed filled him with happiness. In his memoirs [26], Benoît describes his reaction to student comments after his lecture, "Uncanny forms of flattery! Each lifted me to seventh heaven! Truly and deeply, each marked a very sweet day! Let me put it more strongly: it is occasions like that that make my life." For this reason, I believe the classroom is an appropriate stage for a final view of Benoît's work.

In September 2010, a few days after Benoît told me of his diagnosis, I watched the eighty students in my fractal geometry course learn in a single class how to generate the fractals pictured in Figure 12 just by looking at the images and understanding a few attributes of plane transformations.

Their surprise and satisfaction are what Benoît gave me, gave the mathematical world. To those who doubt the value of this approach, I say compare a standard geometry class lesson on plane transformations with this day in any fractals class. The combination of visually complex images and the ability to decode these images by a few simple rules explains why fractals are a wonderful tool for teaching geometry.

A few weeks later in the course, I showed these pictures again and asked the class to find their dimensions. Immediately, they answered $\log (3) / \log (2)$ and $\log (6) / \log (3)$ for the first two, and after a moment, $\log ((-1+\sqrt{3}) / 2) / \log (1 / 2)$ for the third. That thousands, maybe tens of thousands, of students know how to compute and interpret dimensions and that dimension measures complexity and roughness of objects mathematical (Julia sets, Kleinian group limit sets), physical (aggregation clusters, the distribution of galaxies), biological (pulmonary, nervous, and circulatory systems), and artistic (Pollock's drip paintings, at least according to some) are due to Benoît. Some knew bits of the picture; Benoît assembled the whole and got many, many others working on measuring and interpreting dimensions.

For the teacher of a fractals class, the best moment occurs during the day the Mandelbrot set is introduced. The formula $z_{n+1}=z_{n}^{2}+c$ is simplicity itself. Describe the iteration process and the color coding, start the program running (seconds now for images that burned hours or days with the personal computers of the mid1980s), and wait. (See Figure 1.) Startling baroque beauty, but from a class jaded by CGI effects, only a few polite "Oohs" and "Ahhs". Zoom in a few times near the boundary; mysterious spirals of spirals of spirals appear. (See Figure 13). A bit more emphatic exclamations of surprise, and then, "You do remember this is produced by iterating $z_{n+1}=z_{n}^{2}+c$, don't you?" Expressions of disbelief and occasional profanity follow.

Another day or two describing the known geometry of the Mandelbrot set, the arrangement of the cyclic components, the infinite cascade of ever smaller copies of the whole set, and this complicated object starts to seem familiar. Then state the hyperbolicity conjecture and point out it remains a conjecture despite two decades of work by brilliant mathematicians. Beautiful pictures for sure; deep, deep mathematics, you bet. 


\section{Some Key Events in the Life of Benoît B. Mandelbrot}

1924 Born in Warsaw, Poland, 20 November

1936 Moved to Paris

1939 Moved to Tulle

1947 Ingenieur diploma, École Polytechnique

1948 M.S. aeronautics, CalTech

1952 Ph.D. mathematics, University of Paris

1953 Postdoc at MIT, then IAS postdoc of von Neumann

1955 Married Aliette Kagan

1958 Moved to the U.S., joined IBM Thomas J. Watson

1963 Publication of "On the variation of certain speculative prices", [11] and

"The stable Paretian income distribution, when the apparent exponent is near two" [12]

1967 Publication of "How long is the coast of Great Britain?" [13]

1972 Visiting professor of physiology, Albert Einstein College of Medicine

1974 Publication of "Intermittent turbulence in self-similar cascades:

Divergence of high moments and dimension of the carrier" [14]

1975 Publication of Les Objets Fractals: Forme, Hasard et Dimension [15]

1977 Publication of Fractals: Form, Chance, and Dimension [16]

1979 Began studying the Mandelbrot set; formulated the MLC (Mandelbrot set is locally connected) conjecture

1980 Publication of "Fractal aspects of the iteration of $z \rightarrow \lambda z(1-z)$ for complex $\lambda$ and $z "$ [17];

formulated the question that the Mandelbrot set is connected

1982 Publication of The Fractal Geometry of Nature [18];

Fellow of the American Academy of Arts and Sciences;

formulated the $4 / 3$ conjecture and that the inside and outside of the

Brownian boundary curve are statistically self-similar; connectivity of the

Mandelbrot set proved by Douady and Hubbard

1984 TED lecture; formulated the $n^{2}$ conjecture, proved by

Guckenheimer and McGehee

1985 Barnhard Medal, U.S. National Academy of Sciences;

formulated the conjecture that the boundary of the Mandelbrot

set has dimension 2

1986 Franklin Medal, Franklin Institute; D.Sc., Syracuse University

1987 Foreign associate, U.S. National Academy of Sciences;

Abraham Robinson Adjunct Professor of Mathematical Sciences at Yale;

D.Sc., Boston University

1988 Steinmetz Medal, IEEE; Science for Art Prize, Moet-Hennessy-Louis

Vuitton; CalTech Alumni Distinguished Service Award;

Humboldt Preis, Humboldt-Stifftung;

honorary member, United Mine Workers of America;

D.Sc., SUNY Albany, Universität Bremen

1989 Chevalier, National Legion of Honor, Paris;

Harvey Prize for Science and Technology, Technion;

D.Sc., University of Guelph

1990 Fractals and Music, Guggenheim Museum, with Charles Wuorinen

1991 Nevada Prize

1992 D.Sc., University of Dallas

1993 Wolf Prize in Physics;

D.Sc., Union College, Universitè de Franche-Comtè, Universidad

Nacional de Buenos Aires 
1994 Honda Prize; J.-C. Yoccoz awarded the Fields Medal, in part for his work on MLC; Shishikura proved the Mandelbrot set boundary has dimension 2

1995 D.Sc., Tel Aviv University

1996 Médaille de Vermeil de la Ville de Paris

1997 Publication of Fractals and Scaling in Finance [19]

1998 Foreign member, Norwegian Academy of Sciences and Letters; C. McMullen awarded the Fields Medal, in part for his work on MLC; D.Sc., Open University London, University of Business and Commerce Athens

1999 Sterling Professor of Mathematical Sciences at Yale; John Scott Award; publication of Multifractals and 1/f Noise [20]; publication of "A multifractal walk down Wall Street" [21]; D.Sc., University of St. Andrews

2000 Lewis Fry Richardson Award, European Geophysical Society

2001 Member, U.S. National Academy of Sciences; publication of "Scaling in financial prices, I - IV"

2002 Sven Berggren Priset, Swedish Academy of Natural Sciences; William Proctor Prize, Sigma Xi; Medaglia della Prezidenza della Republica Italiana; publication of Gaussian Self-Affinity and Fractals [22] and of Fractals, Graphics, and Mathematics Education [23]; D.Sc., Emory University

2003 Japan Prize for Science and Technology; Best Business Book of the Year Award, Financial Times Deutschland, for The (Mis)Behavior of Markets [25]

2004 Member, American Philosophical Society; publication of Fractals and Chaos. The Mandelbrot Set and Beyond [24], and (with R. Hudson of The (Mis)Behavior of Markets [25]

2005 Sierpinski Prize, Polish Mathematical Society; Casimir Frank Natural Sciences Award, Polish Institute of Arts and Sciences of America; Battelle Fellow, Pacific Northwest Labs; D.CE., Politecnio, Torino

2006 Officer, National Legion of Honor, Paris; Einstein Public Lecture, AMS Annual Meeting; Plenary Lecture, ICM; W. Werner awarded the Fields Medal for proving (with G. Lawler and O. Schramm) the 4/3 conjecture; Doctor of Medicine and Surgery, University degli Studi, Bari, Puglia

2010 D.Sc., Johns Hopkins University; TED lecture; S. Smirnov awarded the Fields Medal for work on percolation theory and SLE related to the $4 / 3$ conjecture.

Died in Cambridge, MA, 14 October

\section{References}

[1] M. F. BARnSLey and S. G. Demko, Iterated function systems and the global construction of fractals, Proc. Roy. Soc. London Ser. A 399 (1985), 243-275.

[2] Michael F. BARnsley, Superfractals: Patterns of Nature, Cambridge University Press, 2006.

[3] Michael F. BARNSLey, Michael Frame (eds.), Glimpses of Benoît B. Mandelbrot (1924-2010), AMS Notices 59 (2012), 1056-1063.

[4] M. V. BERRY, Benefiting from fractals (a tribute to Benoît Mandelbrot), Proc. Symp. Pure Math., vol. 72, Amer. Math. Soc., Providence, RI, 2004, pp. 31-33.

[5] http://www.phy.bris.ac.uk/berry mv/ publications.htmp.

[6] JOHN BROCKMAN, A theory of roughness: a talk with Benoît Mandelbrot (12.19.04), Edge, http://edge. org/conversation/a-theory-of-roughness

[7] J. E. HuTCHINSON, Fractals and self-similarity, Indiana Univ. Math. J. 30 (1981), 713-747.

[8] J. KigAmi, Harmonic calculus on p.c.f. self-similar sets, Trans. Amer. Math. Soc. 335 (1993), 721-755. 
[9] M. L. LAPIDUS, Towards a noncommutative fractal geometry? Laplacians and volume measures on fractals, Contemporary Mathematics, vol. 208, 1997, Amer. Math. Soc., Providence, RI, pp. 211-252.

[10] G. LAWLER, O. SCHRAMM, W. WERnER, The dimension of the planar Brownian frontier is 4/3, Math. Res. Lett. 8 (2001), 401-411.

[11] B. MANDELBROT, The variation of certain speculative prices, J. Business (Chicago) 36 (1963), 394-419.

[12] _ , The stable Paretian income distribution when the apparent exponent is near two, International Economic Review 4 (1963), 111-115.

[13] _ How long is the coastline of Great Britain? Science (New Series) 156 (1967), 636-638.

[14] _ , Intermittent turbulence in self-similar cascades: Divergences of higher moments and dimension of the carrier, J. Fluid Mechanics 62 (1974), 331-358.

[15] _, Les Objets Fractals: Forme, Hasard et Dimension, Flammarion, Paris, 1975.

[16] _ Form, Chance and Dimension, W. H. Freeman, San Francisco, CA, 1977.

[17] , Fractal aspects of the iteration $z-\lambda z(1-z)$ for complex $\lambda$ and $z$, Ann. New York Acad. Sci. 357 (1980), 249-259.

[18] _, The Fractal Geometry of Nature, W. H. Freeman, San Francisco, CA, 1983.

[19] _ Fractals and Scaling in Finance, SpringerVerlag, New York, 1997.

[20] _ Multifractals and 1/f Noise, Springer-Verlag, New York, 1999.

[21] _, A multifractal walk down Wall Street, Scientific American, Feb. 1999, 70-73.

[22] _ Gaussian Self-Affinity and Fractals, SpringerVerlag, New York, 2002.

[23] _ Fractals, Graphics, and Mathematics Education, MAA, Washington, DC, 2002.

[24] _ Fractals and Chaos: The Mandelbrot Set and Beyond, Springer-Verlag, New York, 2004.

[25] _ , The (Mis)Behavior of Markets, with R. Hudson, Basic Books, New York, 2003.

[26] _ The Fractalist: Memoir of a Scientific Maverick, Pantheon, 2012.

[27] D. Mumford, C. SERIES, D. WRIGHT, Indra's Pearls, The Vision of Felix Klein, Oxford University Press, 2002.

[28] JAMES B. BASSINGTHWAighte, LARRY S. LiEBOVITCH, BRUCE J. WEST, Fractal Physiology, Oxford University Press, New York, 1994.

[29] B. J. West, M. BolognA, P. Grigolini, Physics of Fractal Operators, Springer-Verlag, New York, 2003.
MATHFMATICS at the National Security A gency

Make a calculated difference

with what you know.

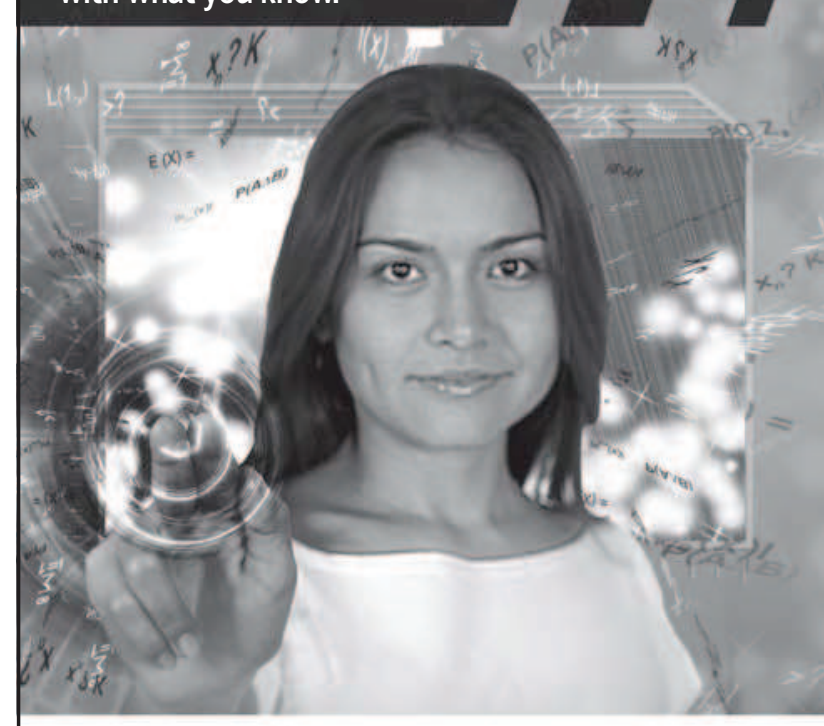

Tackle the coolest problems ever.

You already know that mathematicians like complex challenges. But here's something you may not know.

The National Security Agency is the nation's largest employer of mathematicians. In the beautiful, complex world of mathematics, we identify structure within the chaotic and patterns among the arbitrary.

Work with the finest minds, on the most challenging problems, using the world's most advanced technology.

\section{KNOWINGMATTERS}

\section{Excellent Career Opportunities for Experts in the Following: \\ - Number Theory $\quad$ - Combinatorics \\ - Probability Theory $\quad$ Linear Algebra \\ - Group Theory 》 Plus other opportunities \\ - Finite Field Theory}

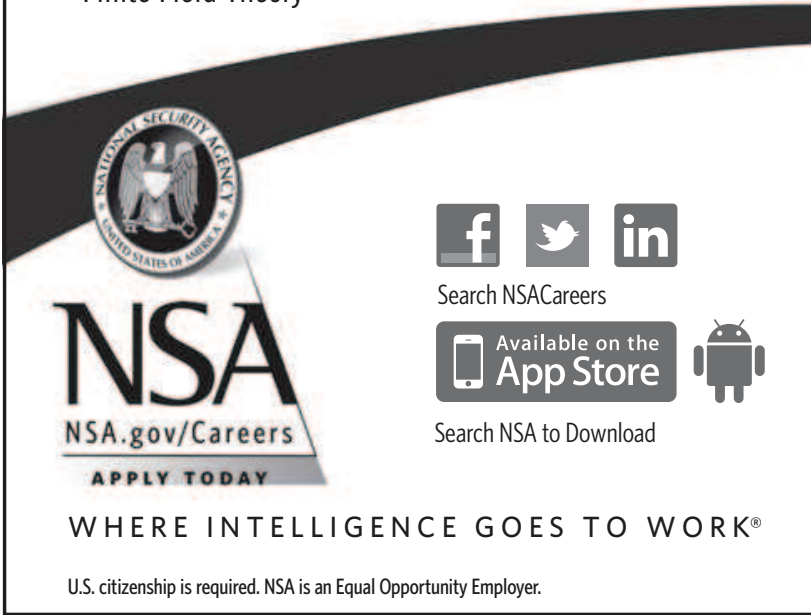

